\title{
When three is a crowd: on keeping outsiders out!
}

\author{
David F. Albertini ${ }^{1}$
}

Published online: 10 May 2015

(C) Springer Science+Business Media New York 2015

Glancing at our cover this month, readers of a scientific or clinical bent will quickly recognize the somewhat artistically rendered triploid human embryo bearing the one too many pronuclei that will spell its fate. Whether left up to the whims of nature, or actions based on the embryologist's day one report, human embryos exhibiting the all-too-familiar signs of three genomes will fail to develop with rare exceptions. Triploids were frequent occurrences in the early days of in vitro fertilization when freshly retrieved oocytes were bathed in sperm-rich fertilization media setting a new bar for effecting the block to polyspermy for oocytes unaccustomed to being so outnumbered by advancing and irrepressible suitors. Our cover model triploid has different origins, however. The keen observer will identify what has become the more recently encountered type of triploid-destined for discard-as the result of ICSI with failure to extrude the second polar body.

No matter the etiology, triploidy is an example of three being a crowd. And, keeping supernumerary sperm out by diverting fertilization strategies towards ICSI in the early 1990s has by and large circumvented any resident deficiencies in mounting the block to polyspermy that would have limited a patient's pregnancy chances in ART times gone by. Very recently, a novel experimental use for human triploids has

Capsule Triploid human embryos have traditionally been viewed as a sign of failure at various levels in the practice of ARTs. But recent studies from China are opening up prospects for testing the feasibility of gene editing in triploid human embryos using CRISPR/Cas9 technology, with preliminary results indicating that molecular genetic interventions of this variety are far from safe or efficient.

David F. Albertini

dalbertini@kumc.edu

1 University of Kansas Medical Center, Kansas, KS, USA gained much attention, launching a debate regarding keeping outsiders out but for very different reasons.

While the rumor mill has been replete with indications of yet another round of genetic engineering broaching the hominid barrier, advances in genome editing of a truly remarkable nature have been emerging in experimental animal and cell culture systems for the past two years. So resounding is the short-term impact of the latest wave of genetic nanosurgery that many organisms whose genetics were intractable with traditional crosses and transgenic manipulations are well within the sights of modeling human disease conditions and someday offering hopes of disease prevention. Leading the way are three types of gene editing tools: clustered regularly interspaced short palindromic repeat (CRISPR)-associated system, zinc finger nucleases (ZFNs), and transcription activator-like effector nucleases (TALENs).

Among the notable, and noteworthy, recent publications is the paper by Liang and colleagues entitled "CRISPR/Cas9mediated gene editing in human tripronuclear zygotes" (Protein Cell DOI 10.1007/s13238-015-0153-5). From an ART perspective, it is important to realize that the triploids used in this study were made the old-fashioned way using IVF and stored in a vitrified state. As indicated, the CRISPR/Cas9 was first employed to target the beta-globin gene, and while the cleavage was accurate, the results show that homologous recombination-based repair was low and most of the edited embryos exhibited mosaicism, a sure sign of variation amongst the targeted pronuclei (which in this case might be expected to be 2 male vs 1 female). Whole exome sequencing in an additional set of experiments further demonstrated offtarget disruptions that acted as repair templates and therefore introduced unwanted mutations in the resultant embryos. Ultimately, these experiments should encourage more research using triploid IVF and ICSI embryos as among the reasons for the failures documented here could be the relative 
accessibility of paternal versus maternal chromatin to the CRISPR/Cas9 components.

The concept of three genomes has hardly escaped attention in the context of the "three parent" embryo debate that has evolved from recent strategies aimed to treat mitochondrial diseases. Readers of JARG need little introduction to matters of mitochondrial aging, spindle transfer, and mitochondrial heteroplasmy, the latter believed to be complicit in the origins of mitochondrial diseases. Various approaches have been suggested deploying young donor egg cytoplasts as spindle recipients or even some form of "youthful" mitochondrial fraction capable of restoring to good health an aged oocyte.

Now comes along a truly creative approach to the problem of mitochondrial manipulations in mammalian oocytes using one of the other gene editing strategies mentioned above. In a recent issue of Cell, the laboratory of Izpisua Belmonte at the Salk Institute reports on the use of TALENs to target and eliminate mutation-bearing mitochondria in mice (see Reddy et al., "Selective elimination of mitochondrial mutations in the germline by genome editing", 2015 161:459-469; doi.org/10. 1016/j.cell.2015.03.051). The importance of studies of this kind are two-fold. First, adapting TALENs to specifically target mitochondrial genomes opens the flood gates for an almost limitless repertoire of components that could seek and complex with DNA or RNA bearing organelles within the limits expected for permeation and involvement of the DNA repair mechanisms that are usurped for purposes of gene editing. Second, by manipulating mitochondrial genomes in oocytes and embryos Reddy and co-workers convincingly demonstrate that offspring so treated pass on these altered genetic traits to their own offspring. Thus, the proofof-concept for a transgenerational impact and cause to contemplate just how many parents at a specific gene locus level could be in the works for future progeny!

Gene editing aside, this month's issue of JARG brings to the forefront a number of studies with immediate relevance to the practice of human ARTs. Among these, and in the category of Technological Innovations, is the study by Ryu and colleagues revealing how micro RNAs are entering the world of non-invasive fetal diagnosis of trisomic conditions. And, extending the debate on PGD is an interesting analysis of embryos at both cleavage and blastocyst stages, adding insight into the genetic gymnastics of human embryos and an important review paper that takes a close look at public perspectives on the use of PGD.

We would finally like our readership to ready themselves for our upcoming issue in June that will focus on the topic of fertilization, and a special issue in July that will cover the latest findings presented at the International Society for Fertility Preservation based on a meeting held earlier this year. Thank you for your support of JARG. 